\title{
Specialis et devota Romanae ecclesiae filia? - Zusammenfassung
}

Zeitlich am Beginn des 13. Jahrhunderts angelangt, soll ein kurzer Ausblick auf den weiteren Verlauf dieses Jahrhunderts der abschließenden Rückschau auf das 12. Jahrhundert vorangehen. Die Frage ist nämlich, wie sich die hier analysierten Entwicklungen langfristig fortsetzten. Danach sind die Ergebnisse der Studie systematisch nach den einleitend vorgestellten und daraufhin bezüglich ihrer Wirkung und ihrem Zusammenspiel im einzelnen Konfliktfall untersuchten päpstlichen Instrumentarien sowie den besonderen, die Beziehungen zwischen dem Papsttum und Toledo prägenden Punkten zusammenzufassen.

Verschafft man sich einen Eindruck von den weiteren Beziehungen zwischen der Toledaner Kirche und dem Papsttum im 13. Jahrhundert, wie sie etwa Peter LineHan ganz allgemein für die iberische Kirche erarbeitet hat, dann kann dieser Eindruck auf den ersten Blick recht ernüchternd sein: Gerade die kastilischen Kirchenmänner sollen etwa die Beschlüsse des IV. Lateranums und die grundsätzliche Verpflichtung des Klerus zur Keuschheit rundheraus abgelehnt haben. Speziell auf den berühmten Erzbischof Rodrigo Jiménez de Rada von Toledo fällt LineHans kritischer Blick, der weder ein Heiliger, noch Asket oder Reformer gewesen sei. Besonders belastend habe sich außerdem der Kreuzzugszwanzigste auf das Verhältnis zwischen der kastilischen Kirche und dem Papsttum ausgewirkt, den die Nachfolger des hl. Petrus jetzt erhoben, da der iberische Episkopat wenig Lust verspürt habe, für die Bestrebungen zur Eroberung des hl. Landes finanziell aufzukommen, während der Feind weiterhin vor der eigenen Haustür stand. Die päpstlichen Geldsammler hätten sich hier nahezu allesamt als ,Diener des Mammons' erwiesen, während die kastilische Kirche tatsächlich allerdings eher wegen der 1247 durch Innozenz IV. den kastilischen Herrschern zugestandenen, tercias reales' finanziell regelrecht ausblutete. Die Synodaltätigkeit in Kastilien sei völlig zum Erliegen gekommen, es sei den päpstlichen Legaten nicht gelungen, die regelmäßige Abhaltung von Provinzialkonzilien durchzusetzen. Überhaupt sei die Herrschaft der kastilischen Könige über ihre Kirchen nahezu absolut gewesen und hätte besonders in der Zeit Alfons' X. von Kastilien (1252-1282) ihren Höhepunkt erreicht. ${ }^{1}$

1 Vgl. Linehan, Church, bes. S. 2f.; S. 16 (Rodrigo Jiménez de Rada „,was no saint, no spartan, no reformer"); S. 52f.; S. 101 (,,servants of Mammon“); zur tatsächlich sehr überschaubaren Anzahl an Diözesansynoden im Bistum Toledo zwischen dem 13. und dem 15. Jahrhundert vgl. etwa José Sánchez Herrero, Los sínodos diocesanos de Toledo 
Was bei LineHans ebenso scharf wie einseitig formulierter Darstellung unterzugehen droht, sind die feststellbaren gegenläufigen Entwicklungen, die er nur am Rand erwähnt. ${ }^{2}$ Gemeint sind damit etwa die offensichtlich in völlige Routine übergegangene Lösung zahlloser Streitfälle in der kastilischen Kirche beispielsweise durch den päpstlichen Legaten Johannes von Abbeville oder auch durch einfache päpstliche nuntii, die weiterhin zunehmende Nutzung der päpstlichen Delegationsgerichtsbarkeit und die grundsätzlich enorme Intensivierung der Korrespondenz zwischen dem Papsttum und der Iberischen Halbinsel. Demetrio Mansilla Reoyo verzeichnet allein für den Pontifikat Innozenz' III. ganze 439 Papsturkunden für die Iberische Halbinsel, das sind nahezu dreieinhalb Mal so viele, wie er für die vielen Jahre zwischen 971 und 1198 vorlegt. Diese Diskrepanz ließe sich noch mit der weitaus besseren Überlieferungssituation nach 1198 erklären. Aber unabhängig von der Registerüberlieferung hat das Kathedralarchiv von Toledo für denselben Papst ganze 24 Originalurkunden katalogisiert. Für den Pontifikat Gregors IX. (1227-1241) liefern die Editionen Santiago Domínguez SÁnchez' dann 999 Stücke und für den nur neun Jahre dauernden Pontifikat Papst Bonifaz' VIII. (1294-1303) ganze 1044 Stücke! $^{3}$ Diese Dimensionen zeigen, dass der iberische Klerus zur päpstlichen Kurie hundert Jahre später in weitaus intensiverem persönlichen Kontakt stand als Ende des 12. Jahrhunderts. Zahlreiche Personen wurden in die direkte Nähe der Päpste platziert, der berühmte Kardinaldiakon Gil de Torres von SS. Cosma e Damiani (1216-1255) und Erzbischof Gonzalo Pérez Gudiel von Toledo (1280-1299), ab 1298 Kardinalbischof von Albano, sind nur zwei willkürlich herausgegriffene Beispiele. ${ }^{4}$ Schon vorher, um 1220, vertrat ein Kleriker namens Bartolomé de Arguiedis speziell die Toledaner Belange an der päpstlichen Kurie, der dann

del siglo XII al XV, in: Stephan Kuttner / Kenneth Pennington (Hgg.), Proceedings of the $5^{\text {th }}$ International Congress of Medieval Canon Law. Salamanca, 21-25 September 1976 (= Monumenta Iuris Canonici, Series C: Subsidia, Bd. 6). Città del Vaticano 1980, S. 93-98.

2 Vgl. etwa die Rezensionen von Joseph F. O'Callaghan, Peter Linehan, The Spanish Church and the Papacy in the Thirteenth Century. (= Cambridge Studies in Medieval Life and Thought. Third Series, Bd. 4) Cambridge 1971, in: Speculum 51 (1976), S. 335f.; Derek W. Lomax, Peter Linehan, The Spanish Church and the Papacy in the Thirteenth Century. (= Cambridge Studies in Medieval Life and Thought. Third Series, Bd. 4) Cambridge 1971, in: the Journal of Ecclesiastical History 24 (1973), S. 66-68; das generelle Urteil von Sмітн, Alexander III, S. 203.

3 Vgl. Domínguez Sánchez (Ed.), Documentos ... de Gregorio IX; Ders. (Ed.), Documentos de Bonicacio VIII (1294-1303) referentes a España.(= Colección Monumenta Hispaniae Pontificia, Bd. 2) León 2006.

4 Zu Gil de Torres vgl. etwa Andreas Fischer, Die Kardinäle von 1216 bis 1304: Zwischen eigenständigem Handeln und päpstlicher Autorität, in: Dendorfer / LütZelschwaB (Hgg.), Kardinalats, S. 155-224, hier S. 216; zu Gonzalo Pérez Gudiel etwa Francisco J. Hernández / Peter Linehan, The Mozarabic Cardinal. The Life and Times of Gonzalo 
wiederum durch einen gewissen Fortunius abgelöst wurde. ${ }^{5}$ Selbst die päpstlichen Geldsammler waren nicht immer erfolglos, und sowohl am IV. Laterankonzil 1215 als auch am II. Konzil von Lyon 1274 nahm eine beachtliche Anzahl Kleriker aus der Kirchenprovinz Toledo teil, wo Gregor X. (1271-1276) wohl in diesem Zusammenhang gegen die kaiserlichen Ansprüche König Alfons' X. von Kastilien gerichtet Rudolf von Habsburg (1218-1291) als römischen König bestätigte. ${ }^{6}$ Vom wieder aufflammenden Primatsstreit, der vorrangig auf der Beweisbasis von Papsturkunden geführt wurde, und der Zunahme kanonistischer Rechtssammlungen während des 13. Jahrhunderts in Toledo war bereits die Rede. Viele der in LineHans Arbeit geschilderten problematischen Entwicklungen reduzierten sich außerdem keineswegs auf das iberisch-päpstliche Verhältnis, sondern sind als gesamteuropäische Phänomene zu betrachten. ${ }^{7}$

Kehrt man damit zurück zum hier behandelten Untersuchungszeitraum, lässt sich zunächst eines feststellen: Integration erweist sich einmal mehr als langwieriger Prozess, in dem auch mit Rückschlägen oder Fehlentwicklungen zu rechnen ist. Das wurde bereits an der Krise zwischen Erzbischof Bernhard von Toledo und dem Papsttum an der Wende vom 11. auf das 12. Jahrhundert deutlich. Stellte man die hier analysierten Entwicklungen graphisch dar, erhielte man schwerlich eine konstant ansteigende Kurve, sondern vielmehr Ausschläge intensiver Kontakte und Durchbruchphasen, wie etwa in den 1160er Jahren, und dazwischen Phasen der Stagnation. Auch wurde nach den hier behandelten rund hundert Jahren im Jahr 1185, wie eben im kurzen Ausblick auf das 13. Jahrhundert angedeutet, kein Abschluss der Integrationsprozesse erreicht, manches intensivierte sich weiter, manches mochte stagnieren oder entwickelte sich möglicherweise sogar zurück. In jedem Fall aber hatten die päpstlichToledaner Beziehungen gegen Ende des 12. Jahrhunderts eine neue Qualität erhalten, die sie in vielen Bereichen fundamental vom Anfang des Jahrhunderts unterschied, und die sich treffend mit den Begriffen „Institutionalisierung“ und „Professionalisierung“ fassen lässt. ${ }^{8}$

Pérez Gudiel. (= Millennio medievale 44, Strumenti e studi N. S., Bd. 5) Florenz 2004; Fischer, Kardinäle, S. 215;

5 Vgl. Linehan, Church, S. 282.

6 Zum IV. Lateranum vgl. etwa Foreville, Lateran I-IV, S. 263-381; García y García, Concilios, S. 451-463; COD 2, S. 227-271; zum II. Konzil von Lyon vgl. COD 2, S. 303-331; aus der Kirchenprovinz Toledo war selbst auf diesem generell eher schlecht besuchten Konzil der Episkopat von Osma, Toledo, Palencia und Segorbe anwesend, vgl. Linehan, Church, S. 212f. mit Anm. 5.

7 Vgl. Dieter Scheler, Rezension zu Peter Linehan, The Spanish Church and the Papacy in the Thirteenth Century. (= Cambridge Studies in Medieval Life and Thought. Third Series, Bd. 4) Cambridge 1971, in: Francia 2 (1974), S. 789-792, hier S. 791f.

8 Herbers, Geschichte des Papsttums, S. 161 in Bezug auf die generelle Entwicklung des Papsttums. 
Für die Empfängerlandschaft Toledo, also Empfänger innerhalb der Diözese Toledo, von den Erzbischöfen bis hin zu einfachen Pfarreimitgliedern, lässt sich für den Verlauf des 12. Jahrhunderts ein enormer zahlenmäßiger Anstieg an Papsturkunden feststellen. Traten zunächst nur Einzelperson als Empfänger auf, so etwa Erzbischof Bernhard, erweitete sich schließlich der Empfängerkreis erheblich. Das heißt, die Anzahl von Personen immer breiterer Schichten der lokalen kirchlichen Hierarchie, die von Toledo aus kostspielige Papsturkunden erwarben und dafür den weiten Weg an den päpstlichen Hof auf sich nahmen, wuchs stetig an, was allein bereits für eine Zunahme bei der Wahrnehmung päpstlicher Autorität seitens der Petenten spricht. Die überwiegende Mehrzahl der ausgestellten Papsturkunden stellen die litterae unterschiedlichster Art dar, Privilegien blieben teuer und relativ selten. Auffällig ist, dass so gut wie keine Korrespondenz mit monastischen Einrichtungen vorliegt. Selbst an den Vorsteher oder die Mönche des päpstlichen Eigenklosters San Servando sind keine direkt adressierten päpstlichen Urkunden bekannt. ${ }^{9}$ Die Überlieferungssituation in den einzelnen Suffraganbistümern gestaltet sich äußerst unterschiedlich. Im Bistum Osma beispielsweise finden sich quasi keine Papsturkunden mehr, während das Bistum Sigüenza aufgrund des dort erhalten gebliebenen Chartularbuchs eine respektable Anzahl solcher Dokumente vorweisen kann, die von der Jahrhundertmitte an stark ansteigt. Zahlreiche Originale dürften dort allerdings den Auseinandersetzungen des 20. Jahrhunderts zum Opfer gefallen sein. Eine ähnliche zeitliche Verteilung der Urkunden weisen die Diözesen Palencia und Segovia auf. Die in wachsender Anzahl erworbenen Papsturkunden entwickelten ihrerseits auch Wirkung auf die lokale Urkundenproduktion. Ein Großteil der Legatenurkunden Erzbischof Bernhards etwa orientiert sich formal wie inhaltlich an der Papsturkunde. Die in ihnen kommunizierten Entscheidungen und Anweisungen werden - wie bei diesem Urkundentypus zu erwarten - ausschließlich durch päpstliche Autorität, nicht etwa durch eine besondere Stellung Toledos legitimiert. Die Anzahl der vorliegenden Toledaner Bischofsurkunden ist relativ gering, die 36 Stücke, die bei Hernández zwischen 1085 und 1185 regestriert sind, zum Großteil Geschäftsunterlagen, liegen in den seltensten Fällen gedruckt vor und konnten hier nur ausnahmsweise berücksichtigt werden. ${ }^{10}$

9 Dieser Befund mag sich durch weitere Archivfunde relativieren, jedoch auch die Aufzeichnungen Paul Fridolin Kenrs und seiner Mitarbeiter aus den Archiven der Iberischen Halbinsel geben keine Hinweise auf intensivere Kontakte zwischen dem Papsttum und Klöstern in der Diözese Toledo.

10 Vgl. Hernández, Cartularios, Nr. 21 (1121); Nr. 39 (1138); Nr. 40 (um 1138); Nr. 41 (Juni 1140); Nr. 48 (Januar 1144); Nr. 52 (17.04.1152); Nr. 60 (Dezember 1147); Nr. 61 (Januar 1148); Nr. 65 (Juli 1149); Nr. 72 (März 1150); Nr. 75 (Mai 1150); Nr. 80 (Juli 1150); Nr. 85 (Januar 1152); Nr. 87 (Februar 1152); Nr. 95 (August 1154); Nr. 96 (August / September 1154); Nr. 97 (September 1154); Nr. 98 (September 1154); Nr. 109 
Die Urkunde Cerebruns von Toledo aus dem Jahr 1176 etwa, die die Verlegung der Diözese Segobrica nach Albarracín festhält, weist besonders bei der Form der Datierung durchaus mögliche Einflüsse der Papsturkunde auf. Schließlich wirkte die Papsturkunde sogar auf die königliche Urkundenproduktion. In der Übernahme des Bleisiegels und der Urkundenform des Mandats folgte die Kanzlei Alfons' VIII. von Kastilien ganz offensichtlich dem päpstlichen Vorbild. Der Großteil der besagten päpstlichen Privilegien wurde im Zuge des Primatsstreits zwischen 1143 und 1179 erworben, als die Bedeutung päpstlicher Privilegierung für die Toledaner Primatswürde stark anstieg. Die Betonung der Herkunft der Toledaner Primatswürde aus der westgotischen Vergangenheit wurde in den Papstprivilegien von 1118 bis 1156 dagegen stetig zurückgedrängt und spielte im Primatsstreit wenigstens bis zum anzunehmenden Prozess in den Jahren 1166/1169 zwischen Toledo und Tarragona nur noch eine untergeordnete Rolle. Zwar wurde nach der ebenfalls anzunehmenden Vorlage der Exceptio de dignitate Toletane ecclesie in ihrer frühen Redaktionsstufe bei Alexander III. päpstlicherseits wieder häufiger darauf verwiesen, es bestand jedoch nun kein Zweifel mehr, dass der Toledaner Primat vor allem anderen durch die zahllosen päpstlichen Privilegien legitimiert wurde. Neben die Primatsprivilegien traten die insgesamt sieben vorliegenden päpstlichen Besitz- und Grenzbestätigungen (1099, 1127, 1148, 1156, 1161, 1187 und 1192). Im Jahr 1148 wurde ein besonderer päpstlicher Schutzpassus in diese Privilegien aufgenommen, der womöglich ein Stück weit ersetzte, dass sich auf der Iberischen Halbinsel einzig das Königreich Kastilien-León nicht in ein Schutzverhältnis zum Papsttum begeben hatte. Die beiden Privilegien von 1187 und 1192 vereinen schließlich Primats- und Besitzbestätigung, die Bedeutung der Toledaner Primatswürde wurde allein dadurch deutlich zurückgestellt. Eigene Pallienprivilegien an die Erzbischöfe von Toledo sind aus dem ausgehenden 11. und dem 12. Jahrhundert nicht bekannt, statt dessen wurde das Pallium bis dahin meist im Zusammenhang mit der Bestätigung des Toledaner Primats gewährt. Gegen Ende des 12. Jahrhunderts erwarben sogar Toledaner Pfarreikleriker Privilegien, indem sie sich von Alexander III. und Lucius III. königlicherseits zugestandene Schutz- und Freiheitsrechte bestätigen ließen. Durch Toledaner Petenten erworbene päpstliche Mandate nahmen gerade während des Primatsstreits enorm zu und übertrafen zahlenmäßig selbst jene Anfang des 12. Jahrhunderts gegen Erzbischof Bernhard von Toledo erworbenen Mandate. Die gestiegene Bedeutung von Papsturkunden wurde sogar an

(November 1155); Nr. 119 (Mai 1157); Nr. 126 (Dezember 1159); Nr. 127 (Dezember 1159); Nr. 134 (11.03.1162); Nr. 135 (März 1162); Nr. 143 (30.11.1164); Nr. 144 (Dezember 1164); Nr. 147 (1152-1166); Nr. 160 (1171); Nr. 165 (März 1174); Nr. 166 (März 1174); Nr. 174 (01.03.1176); Nr. 178 (1176); Nr. 182 (20.12.1177); Nr. 183 (August 1178); Nr. 185 (01.12.1178); Nr. 204 (07.12.1183). 
der Toledaner Fälschertätigkeit sichtbar: War es um die Jahrhundertwende noch die westgotische Vergangenheit, die derartig bedeutsam erschien, dass auf sie etwa in Form der Divisio Wambae gefälscht wurde, erschlich die Toledaner Seite womöglich 1123 ein Papstprivileg, das Erzbischof Bernhard die päpstliche Legationswürde über die gesamte Iberische Halbinsel bestätigte. Und auch in den gefälschten Passagen der Exceptio de dignitate Toletane ecclesie spielte das Papsttum eine entscheidende Rolle.

Zum kanonischen Recht ist festzustellen, dass sich zu Beginn des 12. Jahrhunderts in der Kirchenprovinz Toledo im Gegensatz zu anderen Regionen der Iberischen Halbinsel keine kanonistische Sammlung der Reformzeit nachweisen lässt. In Form der Collectio Hispana lag bereits eigenes, genuin iberisches Kirchenrecht in kodifizierter Form vor, durch das sich weitreichende kirchenpolitische Vorrechte einfordern ließen, die dem Primas von Toledo kurz vor dem Untergang des Westgotenreichs zugesprochen worden waren. Erzbischof Bernhard waren Sammlungen aus dem Norden der Pyrenäen, wie die pseudoisidorischen Dekretalen, allerdings durchaus bekannt und er setzte deren Kenntnis auch bei anderen Prälaten, wie dem Erzbischof von Tarragona, voraus. Dass andere kanonistische Sammlungen auf der Iberischen Halbinsel, wie etwa die Collectio Caesaraugustana, eine dezidiert antiprimatiale Stoßrichtung aufwiesen, legt die Vermutung nahe, man habe sich in Toledo zu Beginn des 12. Jahrhunderts lieber auf besagte, die eigenen Ansprüche beinhaltende Collectio Hispana beschränkt, während die Gegner Toledos die neuen Sammlungen aus dem Norden der Pyrenäen gezielt gegen den Toledaner Primat zu Rate zogen. Ein Wandel setzte zur Jahrhundertmitte ein, als sich die päpstliche delegierte Gerichtsbarkeit in der Kirchenprovinz Toledo verbreitete und damit eine Ausbreitung des römisch-kanonischen Prozessrechts einherging: Streitfälle belegen nun auch hier die Nutzung von Kirchenrecht aus dem Norden der Pyrenäen und deuten etwa auf die Anwendung des durch den Kardinallegaten Hyacinth und seine rechtsgelehrten Begleiter auf die Halbinsel verbrachten Decretum Gratiani hin. In Toledo oder in der Schreibstube eines Suffraganbistums wurde zwischen 1166 und 1169 die erste Redaktionsstufe jener wichtigen namenlosen Rechtssammlung, deren prominentester Teil als Appendix Seguntina bekannt ist, beendet. Erste Kompilationsarbeiten dürften in Poitiers wahrscheinlich um 1148 durch die neue Klerikergeneration der Kirchenprovinz Toledo unternommen worden sein, welche dorthin teilweise verwandtschaftliche Beziehungen unterhielt, und den Regularkanonikern oder zumindest einem dieser Bewegung aufgeschlossenen Umfeld entstammte, wie etwa Cerebrun, später Bischof von Sigüenza und schließlich Erzbischof von Toledo. Vorliegende Sammlung stellt die einzige bekannte vorgratianische Rechtssammlung dar, die in Kastilien fertiggestellt worden sein soll. Diverse, besonders im späteren Frankreich weit 
verbreite Rechtstexte, so etwa die Panormia, Burchards bzw. Ivos Decretum oder der Polycarpus dienten als Vorlagen. Sogar speziell zur Primatsfrage wurden aus der Panormia für die Toledaner Position hoch brisante Texte übernommen, die die Bedeutung des Papstes für die Primatswürde unterstrichen, die vorchristliche Tradition eines Primatssitzes, wie sie aus Pseudoisidor bekannt war, unterschlugen und die primatialen Rechte enorm einschränkten. Diese Passagen dürften die Vorlage für die Exceptio de dignitate Toletane ecclesie dargestellt haben, die sich wiederum als exakte Antwort auf die aus der Panormia übernommenen Passagen zum Primat lesen lässt und womöglich am Gericht Alexanders III. in einem für Toledo schließlich erfolgreichen Prozess gegen Tarragona als Prozesseinrede eingesetzt wurde. Die Sammlung beinhaltet außerdem die Kanones des Provinzialkonzils von Segovia 1166, als die selbständige Herrschaft Alfons' VIII. von Kastilien eine päpstliche Geburtshilfe erfuhr, und diverse weitere Texte päpstlich geleiteter Konzilien und Dekretalen, die die päpstliche Autorität betonen. Die gesamte Sammlung lässt sich als hervorragendes Distributionsinstrument päpstlicher Vorstellungen werten, besonders da sie vor Ort offenbar eine breite Nutzung erfuhr. Zunehmende Rechtsnachfragen in Rom, die päpstliche Dekretalen hervorbrachten, sprechen zusätzlich für die sich im Untersuchungsraum ausbreitende Akzeptanz des päpstlichen Gerichtsprimats. Der Erwerb solcher Schreiben und ihre Aufnahme in die nachgratianischen Dekretalensammlungen sorgten für eine Angleichung der kirchenrechtlichen Praxis im Rezeptionsradius. Zwar liegen im Untersuchungsraum keine bedeutenden Sammlungen von solchem ius novum aus dem Norden der Pyrenäen, durch das päpstliche Dekretalen endgültig Konzilsbeschlüssen vorangestellt wurden, für den hier relevanten Zeitraum vor, mit der Collectio Seguntina findet sich in der Kirchenprovinz Toledo Ende des 12. Jahrhunderts jedoch immerhin die bedeutendste lokal gebräuchliche Dekretalensammlung dieser Zeit. Von der Jahrhundertmitte an lässt sich also in der Kirchenprovinz Toledo ein bedarfsbedingtes Aufholen der Verspätung auf kirchenrechtlichem Gebiet feststellen. Das römisch-kanonische Recht stieß nun auf breites Interesse und ausgiebige Nutzung, wenngleich das alte westgotische Recht in Form des ,fuero juzgo ' besonders im privatrechtlichen Bereich weiterhin genutzt wurde. Auch an der Karriere von Persönlichkeiten wie Arderico von Mailand oder Pedro de Cardona lässt sich dieses Interesse belegen. Am Ende des Jahrhunderts nutzten nachweislich selbst die Rechtsvertreter des einfachen Toledaner Pfarreiklerus kirchenrechtliches Schriftgut. In Toledo sind schließlich seit Beginn des 13. Jahrhunderts zahlreiche kirchenrechtliche Sammlungen von jenseits der Pyrenäen bekannt, das allein relativiert LineHANs negativen Befund bezüglich der generellen Reformfreudigkeit des Toledaner Klerus doch stark.

Schon rund 20 Jahre vor der Eroberung Toledos 1085 bereisten päpstliche Legaten den Westen der Iberischen Halbinsel und sorgten für das Bekannt- 
werden päpstlicher Vorstellungen und Ziele. Bereits diese ersten Kontaktaufnahmen zu einer Gegend, in der das Papsttum bis dahin weitgehend vergessen worden war, erwies sich stellenweise als relativ wirkungsvoll, besonders beim Wechsel vom altspanischen zum römischen Ritus. So darf der päpstliche Kardinallegat Richard von Marseille wohl auch für die päpstliche Ablehnung des ersten, heute unbekannten Kandidaten für den Erzstuhl von Toledo verantwortlich gemacht werden. Ebenso dürfte er bei der Designation Bernhards, des Abtes von Sahagún, zum Erzbischof von Toledo anwesend gewesen sein. Besonders Gregor VII. ist für die Ernennung ständiger päpstlicher Legaten bekannt, aber erst Urban II. nutzte dieses Mittel aufgrund momentaner Handlungseinschränkungen und des Ungehorsams Tarragonas gegenüber der Toledaner Primatswürde und ernannte Erzbischof Bernhard zum ständigen päpstlichen Legaten für die Iberische Halbinsel und die Kirchenprovinz Narbonne. Die Ansammlung diverser Befugnisse als Primas, Legat und provisorischer Metropolit über alle iberischen Diözesen, deren zuständige Metropole noch nicht wiedererrichtet wurde, ließen Erzbischof Bernhard offensichtlich in der Wahrnehmung vieler iberischer Prälaten als papstähnliche Figur erscheinen. Dies führte zu diversen Appellationen bei den Päpsten. Die Quellen zeichnen ein ambivalentes Bild von Erzbischof Bernhard in den ersten beiden Jahrzehnten seines Pontifikats. Die Versuche, seinen Einfluss auf die west- und nordiberische Kirche auszudehnen - am frühesten und erfolgreichsten entzog sich ihm die ostiberische Kirche, von einem Tätigwerden nördlich der Pyrenäen ist nichts bekannt - muss aus der speziellen Toledaner Situation heraus gesehen werden. Es existierte schließlich eine enorme Diskrepanz zwischen den zahlreichen wohlklingenden Titeln und der reellen Machtgrundlage des Toledaners, da über die Eroberung Toledos hinaus zunächst weitere Erfolge in der Reconquista ausblieben. Andererseits trat Erzbischof Bernhard, wie sich seinen Legatenurkunden entnehmen lässt, durchaus als päpstlicher Amtsvertreter auf. Während des Pontifikats Urbans II. war Erzbischof Bernhard zwischen 1093 und 1100 einziger päpstlicher Legat auf der Iberischen Halbinsel. Zu Rainer von S. Clemente hatten sich die Beziehungen wahrscheinlich bereits deshalb getrübt, weil es dem katalanischen Klerus mit seiner Unterstützung gelungen war, Erzbischof Bernhard aus dem Restaurationsprozess Tarragonas herauszudrängen. Auf den Papstthron gewählt entsandte Paschalis II. einen der größten Gegner Erzbischof Bernhards, Richard von Marseille, als ersten päpstlichen Legaten des jungen 12. Jahrhunderts auf die Iberische Halbinsel. Entsprechend schlecht gestaltete sich das Zusammenwirken der beiden päpstlichen Amtsträger, des ständigen und des ortsfremden Legaten. Weder für den Besuch des Kardinallegaten Richard noch für die Legationsreise Abt Pontius' von Cluny lässt sich eine Kooperation auch nur erahnen, vielmehr waren die Verhältnisse offenbar durch Konkurrenz, unversöhnliche Haltungen, di- 
vergierende Interessen oder schlichtweg durch gegenseitiges Nichtbeachten geprägt. Die Eindrücke der ortsfremden Legaten dürften wenig dazu beigetragen haben, die an sich bereits wenig positive Meinung Paschalis' II. über Erzbischof Bernhard zu revidieren. Ein feststellbarer Wandel trat erst mit den Legationsreisen der Kardinallegaten Boso und Deusdedit ein, zu denen Bernhard nun wohl ganz gezielt den Kontakt suchte, mit denen er kooperierte, über die er sich offensichtlich bemühte, der Krise mit der kirchlichen Zentrale zu begegnen, und schließlich auch einige Erfolge verbuchen konnte. Die Reduzierung des Toledaner Legationsbezirks durch Paschalis II. und die Ernennung Diego Gelmírez' von Compostela zum ständigen Legaten über Mérida / Compostela und Braga durch Calixt II. beschränkten allerdings den Wirkungsradius der Toledaner Legationswürde weitgehend auf die eigene Kirchenprovinz und führten den Titel damit im Grunde ad absurdum. Der Beginn eines lähmenden Streits um die ständige Legationswürde über die gesamte Iberische Halbinsel, der von beiden Seiten mit Fälschungen geführt wurde, brachte alle beiden Konkurrenten häufiger denn je an den päpstlichen Hof. Das System der ortsfremden päpstlichen Legaten funktionierte jedoch mittlerweile derartig erfolgreich, dass sich das System der ständigen Legation überlebt hatte und 1130 womöglich durch den Kardinallegaten Humbert von S. Clemente in Kastilien-León gezielt beendet wurde. Deshalb ist aus päpstlicher Sicht die ständige Legation des Toledaner Erzbischofs aber nicht etwa als völliger Fehlschlag zu sehen. Ein Nebeneffekt dieser Auseinandersetzungen bestand nämlich in der Verbreitung der Vorstellungen, dass überprovinziale Konzilien der Genehmigung des Papstes und der Leitung eines päpstlichen Legaten bedurften. Diese hatten sich in den 30er Jahren des 12. Jahrhunderts in Kastilien-León bereits derart fest etabliert, dass auch der selbsternannte Kaiser Alfons VII. den Papst regelmäßig um die Entsendung päpstlicher Legaten bat, um gemeinsam mit diesen auf Konzilien Probleme zu lösen. Bis 1172 war der Episkopat und wahrscheinlich auch ein Teil des höheren Klerus aus der Kirchenprovinz Toledo auf Legatenkonzilien präsent, wo nicht nur einige für die Provinz relevante Rechtsentscheidungen getroffen, sondern auch die kirchenreformerischen Prinzipien und Ziele verbreitet wurden. Die Entscheidungen des II. Lateranums von 1139 etwa wurden auf dem Legatenkonzil von Vallaldolid 1143 für den lokalen Klerus wiederholt. Wenn allerdings ortsfremde päpstliche Legaten die Iberische Halbinsel aufsuchten, durchquerten sie die Kirchenprovinz Toledo höchstens in deren nördlichsten Ausläufern, Visitationsreisen durch die Provinz sind nicht bekannt. Dass sich ortsfremde päpstliche Legaten in der Kirchenprovinz Toledo allenfalls nach Valladolid oder Carrión de los Condes wagten, dürfte vor allem mit der militärischen Lage zu erklären sein. Die Ausnahme stellte auch hier der päpstliche Kardinallegat Hyacinth von S. Maria in Cosmedin dar. Sein erster Besuch 1154/1155 auf der 
Iberischen Halbinsel wies dabei allerdings noch wenige Besonderheiten auf. Sein Kommen als neuer und unvoreingenommener päpstlicher Legat wurde wohl von Alfons VII. vom Papsttum erbeten und Hyacinth erfuhr zunächst eine zuvorkommende Aufnahme. Zwar sorgte Hyacinth für die Verbreitung römischkanonischen Rechts und nahm sich auch im Untersuchungsraum einiger Streitgegenstände an, gerade in Kastilien hatte er aber gegenüber dem Bischof von Osma, gegenüber den Kanonikern von Valladolid und in der Primatsfrage, deren Klärung ihm erst nachträglich aufgetragen worden war, doch einige Niederlagen einzustecken. Die Primatsfrage hatte eindeutig hinter dem Kampf gegen die Almohaden zurückzustehen. Trotzdem kam es bei dieser Gelegenheit zu den ersten Begegnungen zwischen dem Legaten und Erzbischof Johannes von Toledo, auf die hin sich Hyacinth zurück am päpstlichen Hof für den Toledaner Primat einsetzte. Erstmals 1172 lässt sich mit dem Besuch Hyacinths ein päpstlicher Kardinallegat direkt in Toledo belegen. Auf seiner zweiten Spanienreise entfaltete der Legat auch unmittelbar vor Ort eine rege Tätigkeit, von der sogar einfache Landpfarrer profitierten. Die Wirkung, die von der Präsenz des päpstlichen Alter Ego in Toledo ausging, war offenbar enorm. Sein Besuch stellte wohl einen entscheidenden Auslöser dafür dar, dass immer breitere Kreise des Toledaner Klerus auf die Appellation beim Papsttum zurückgriffen. Aber auch umgekehrt schien womöglich gerade die Anwesenheit Hyacinths in Toledo und bei der Belagerung von La Huete auf den Legaten große Wirkung gehabt zu haben. Seine Legationsreise steht für einen Wendepunkt in der päpstlichen Reconquistapolitik. Noch vor Ort förderte der Legat den Kampf gegen die Almohaden, etwa durch die Bestätigung des Santiagoordens, und seit seiner Rückkehr 1175 forderte das Papsttum nun für den Rest des Jahrhunderts die Sammlung aller christlichen Kräfte der Iberischen Halbinsel gegen al-Andalus. Toledo wurde in dieser Politik, die tatsächlich seit dem Tod Gregors VII. nun wieder Züge päpstlichen Tätigwerdens motu proprio erkennen lässt, mehr und mehr zur Koordinations- und Schaltstelle dieser Bemühungen. Auf einem gesamtiberischen Konzil unter Toledaner Leitung sollten die Ursachen der Feindseligkeiten unter den ,cinco reinos" gesammelt werden, um sie dem Papst und seinen Kardinälen zur Lösung vorzulegen - eine idealistische Idee, die wie so viele andere Einigungsversuche der christlichen Reiche an den Realitäten der Zeit scheiterte. Kardinal Hyacinth war an der päpstlichen Kurie zum Spezialisten für iberische Fragen geworden und darf als Schlüsselfigur für die päpstlich-Toledaner Beziehungen und die Integration des Untersuchungsraums gelten. Von der Jahrhundertmitte an lässt sich außerdem ein gewisser Wandel in der päpstlichen Legationspolitik erkennen. Die Reisen der Kardinallegaten sind, was Entscheidungen in der Kirchenprovinz Toledo betrifft, weitaus besser dokumentiert, die Legaten verweilten länger, dafür ging die Anzahl ihrer Besuche zurück. Zwischen 1150 und 
1197 besuchten nur Hyacinth und sein Neffe auf jeweils zwei ausgedehnten Reisen auch die Kirchenprovinz Toledo. Dieser Befund lässt sich auf die gesamte Halbinsel übertragen. Claudia ZEY sucht die Gründe am päpstlichen Hof selbst und nicht auf der Iberischen Halbinsel: So nennt sie vor allem die personellen Diskontinuitäten, die die zahlreichen Pontifikatswechsel nach 1143 mit sich brachten und weist außerdem darauf hin, wie die Etablierung eines reichen Wissens- und Erfahrungsschatzes zur Iberischen Halbinsel - besonders in Gestalt Hyacinths - die Entsendung weiterer Kardinäle überflüssig machte. ${ }^{11}$ Dabei darf allerdings nicht übersehen werden, dass die Anzahl der Legationsreisen hierarchisch niedriger stehender päpstlicher Kleriker anstieg, deren Tätigkeiten zwar weitaus schlechter dokumentiert sind als die der Kardinäle, die jedoch deshalb nicht weniger bedeutsam waren. Auf die Reisen des päpstlichen Subdiakons Petrus etwa wurde ebenso verwiesen wie auf die des päpstlichen Notars Michael. Sie sammelten Gelder für die päpstlichen Kassen und wurden als Fachleute in speziellen Streitfragen tätig. Dass das Papsttum durchaus ein Interesse besaß, seine eigenen Leute als Ansprechpartner in Toledo zu installieren, darauf deuten die Bitten um die Aufnahme Michaels und seines Begleiters Johannes ins Toledaner Kathedralkapitel. Sowohl die krisenhaften Entwicklungen zur Jahrhundertwende als auch die später engen Toledaner Beziehungen zu Hyacinth machen eines vollkommen klar: Wie niemand anderes prägten die Legaten die päpstliche Haltung gegenüber der entsprechenden Region.

$\mathrm{Zu}$ einer feststellbaren Verzögerung kam es bei der Verbreitung der päpstlichen Delegationsgerichtsbarkeit in der Kirchenprovinz Toledo. Dabei waren Erzbischof Bernhard von Toledo schon vor der Wende zum 12. Jahrhundert vom Papsttum richterliche Aufgaben übertragen worden. Wegen der Unmöglichkeit, die einzelnen Aufträge bzw. Tätigkeiten dieses Prälaten einem ganz bestimmten Amt zuzuweisen, ist es kaum mit Sicherheit zu entscheiden, ob Bernhard als Primas, als (provisorischer) Metropolit, als Legat oder etwa bereits als päpstlicher delegierter Richter zum richterlichen Tätigwerden aufgefordert wurde. In immerhin einer überlieferten päpstlichen Kommissorie finden sich keinerlei Nennungen anderer Titel als die eines Erzbischofs von Toledo, was auf die Ernennung Bernhards zum päpstlichen delegierten Richter hinweisen kann. Bei zahlreichen Gelegenheiten wurde der Toledaner Prälat allerdings nicht als Richter akzeptiert, zu tief war er häufig selbst in die jeweiligen Streitigkeiten involviert, oder es konnte ihm aus anderen Gründen die notwendige Unvoreingenommenheit abgesprochen werden. Selten sprach Erzbischof Bernhard überhaupt ein Urteil, in keinem bekannten Fall wurde dieses befolgt. Der Toledaner Richter blieb viel eher untätig oder zog sich aus dem entsprechenden

11 Vgl. ZeY, Möglichkeiten. 
Fall zurück, hatte überdies Grund zur Resignation aufgrund mangelnder päpstlicher Rückendeckung. Diese Entwicklungen verschärften die besagte Krise der Jahrhundertwende und bewirkten offenbar, dass die päpstliche Delegationsgerichtsbarkeit in der Kirchenprovinz Toledo selbst jahrzehntelang keine Rolle spielte und auch Erzbischof Bernhard im letzten Jahrzehnt seines Pontifikats nicht mehr mit richterlichen Aufgaben betraut wurde. Erst ab der Jahrhundertmitte und sicherlich gefördert durch die häufige Präsenz Toledaner Prälaten an der päpstlichen Kurie aufgrund des Primatsstreits - welcher letztendlich auch ein Rechtsstreit war, der am päpstlichen Gericht ausgefochten werden sollte - nehmen päpstliche Kommissorien an den Untersuchungsraum schlagartig erheblich zu. Der Kontrast zwischen den Konflikten um die Verwaltungshoheit über das Kloster San Servando etwa und den Auseinandersetzungen um die Toledaner Klerikerverbrüderungen könnte kaum größer sein. Die Prälaten der Kirchenprovinz Toledo entdeckten das Instrumentarium für sich und auch die Erzbischöfe von Toledo wurden wieder von anderen Kirchenmännern als delegierte Richter vorgeschlagen. Dadurch wurde die Verbreitung des römischkanonischen Prozessrechts vor allem durch die häufige praktische Anwendung gefördert. Einige Kommissorien beinhalten noch ganz detaillierte und konkrete Handlungsanweisungen, andere setzten das formal korrekte Handeln der Empfänger bereits als selbstverständlich voraus. Die Möglichkeit der Appellation an den Papst als obersten Schiedsrichter und Gerichtsherrn wurde damit innerhalb von ein bis zwei Jahrzehnten in der gesamten Kirchenprovinz bekannt. Schnell nutzten dieses Mittel nicht nur Erzbischöfe oder Bischöfe, sondern auch die Mitglieder der Kathedralkapitel und schließlich sogar einfache Gemeindepfarrer. Dabei wurden die Urteile der delegierten Richter im Untersuchungsraum nicht etwa gehorsamer befolgt als anderswo, was etwa im Prozess Sigüenzas gegen Osma offensichtlich wurde. Die delegierten Richter begegneten denselben Schwierigkeiten wie in anderen Teilen des orbis christianus auch, Ausreden, Prozessverschleppung, Kontumanz und zahlreichen anderen Hemmnissen. Wie neu und ungewohnt dieses Instrumentarium auch für die königlichen Gewalten war, wurde ebenfalls im Streit zwischen Osma und Sigüenza an der Reaktion des Königs von León deutlich. Aber auch in der Kirchenprovinz Toledo sorgte die verbreitete Nutzung der päpstlichen Delegationsgerichtsbarkeit schnell für eine enorme „Verdichtung der Kommunikation“12 zwischen Untersuchungsraum und päpstlicher Zentrale, für die Ausbreitung universaler Rechtsvorstellungen, für die Angleichung von Prozessverfahren und Rechtsgrundlagen. Die schnelle und weite Verbreitung der päpstlichen Delegationsgerichtsbarkeit und die daraus resultierenden Folgen zählen zweifellos zu den wichtigsten Fortschritten im In-

12 MüLler, Entscheidung, S. 117. 
tegrationsprozess. Wenn selbst der normale Stadtpfarrer die erfolgversprechende Möglichkeit sah, gegen seine kirchlichen und weltlichen Oberen am päpstlichen Gericht zu klagen und dort einen zwar kosten- und zeitintensiven, jedoch auch streng reglementierten, berechenbaren und fairen Prozess anstrengen zu können, spricht das für eine breite Akzeptanz päpstlicher Autorität in Form des päpstlichen Gerichtsprimats. Auch in der Kirchenprovinz Toledo reifte die päpstliche Delegationsgerichtsbarkeit damit ,zu einem System päpstlicher Fernwirkung, das die administrative Effizienz der Kurie stärkt[e]““. ${ }^{13}$

Einen enormen Wandel erfuhr außerdem die konziliare Praxis des kastilischen Klerus. Das IV. Konzil von Toledo (633) hatte die Abhaltung von Reichskonzilien festgeschrieben, die vorher wie nachher bevorzugt in Toledo abgehalten worden waren. Nicht zuletzt darauf fußte die Stellung Toledos als Sitz des Primas der westgotischen Kirche, obwohl hier weniger die Metropoliten von Toledo, als vielmehr die westgotischen Könige eine wichtige Leitungsfunktion eingenommen hatten. Zu Beginn des 11. Jahrhunderts gab es auf der Iberischen Halbinsel zwar keinen Primas mehr, und die einstige westgotische Einheit war dahin, aber in León 1017 etwa, oder in Coyanza 1055 saßen diesen gemischt kirchlich-weltlichen Versammlungen unbestritten die Könige vor. Am Ende des 11. Jahrhunderts fanden die weiterhin stark durch den König dominierten Reichskonzilien dann regelmäßig in Präsenz eines päpstlichen Legaten statt, der auch dort die Prinzipien der Kirchenreform, auch was die konziliare Praxis betraf, publik gemacht haben dürfte. Gewisse Parallelen zur westgotischen Vergangenheit sind unübersehbar, als daraufhin in den ersten beiden Jahrzehnten des 12. Jahrhunderts der ständige apostolische Legat und Primas der gesamten Iberischen Halbinsel, Erzbischof Bernhard von Toledo, gemeinsam mit den Herrschern Kastilien-Leóns Konzilien mit häufig reichsweiter Teilnahme abhielt, die aufgrund Bernhards Titels eines päpstlichen Legaten kirchenrechtlich allerdings weitgehend unbedenklich waren. Die wenigen Quellen, die zu diesen Versammlungen vorliegen, liefern außerdem keinerlei Hinweise drauf, dass dort explizit an das westgotische Erbe angeknüpft worden wäre. Die Legatenurkunden Erzbischof Bernhards präsentieren den Prälaten vielmehr als päpstlichen Amtsträger, der für die Verbreitung kirchenrechtlcher Vorstellungen zur Konzilienpraxis sorgte, die sich selbst noch im Prozess der Ausdifferenzierung befanden. Der Legationsstreit zwischen Toledo und Tarragona und der weitaus besser dokumentierte zwischen Toledo und Compostela entzündete sich vor allem an der Frage nach dem Recht auf Einberufung und Leitung von Konzilien und zeigt die weite Verbreitung der kirchenrechtlichen Vorstellungen in Kastilien-León, dass überprovinziale Konzilien vom Papsttum zu genehmigen und durch einen

13 MüLler, Gesandte, S. 64. 
päpstlichen Legaten zu leiten seien. Zwischen 1130 und 1158 trat die konziliare Praxis wieder in eine neue Phase: Die Versammlungen der ständigen päpstlichen Legaten wurden abgelöst durch Konzilien, denen Kardinallegaten a latere des Papstes vorsaßen. Die wichtige Stellung des weltlichen Herrschers wurde zwar längst nicht gänzlich abgeschafft, es war jedoch wohlgemerkt der König, der in den meisten Fällen die Entsendung eines päpstlichen Kardinallegaten a latere erbat. Die Rückkehr zu einer Konzilspraxis, die gänzlich auf die Anwesenheit eines päpstlichen Legaten und damit auf päpstliche Genehmigung und Kontrolle verzichtete, war nicht mehr möglich. Der Primas von Toledo spielte bei allen diesen Legatenkonzilien keine wirkliche Rolle, ,Primatskonzilien“ hat es im 12. Jahrhundert nie gegeben. Dass der Toledaner Primas anders als etwa der von Vienne nicht zur Abhaltung überprovinzialer Konzilien berechtigt war, schien weitgehend unbestritten. Wegen der vielen Divergenzen des kastilischen Klerus und der zentrifugalen politischen Entwicklungen wurden solche Legatenkonzilien mit breiter iberischer Beteiligung immer seltener. Ohnehin hatten die Legaten in den ostiberischen Herrschaften, in Aragón, Navarra und Katalonien, stets eigene Konzilien abgehalten. Nach dem Zerbrechen der kastilisch-leonesischen Einheit deckten sich viele Königreiche beinahe mit einer Kirchenprovinz. Weiterhin verblieben die meisten Reichsprälaten am Hof des Königs. Das erschwert die Erforschung der konziliaren Tätigkeit, da im Einzelfall aus der Unterschriftenliste eines königlichen Schriftstücks nicht zu entscheiden ist, ob zur Beschlussfindung eine kirchliche Versammlung unter Anwesenheit aller Unterzeichnenden anberaumt, oder die Urkunde stattdessen nur nach und nach den Unterzeichnern zur Bestätigung vorgelegt worden war. Tragfähige Nachrichten über regelmäßige Provinzialkonzilien existieren für Kastilien nicht, das wichtige Konzil von Segovia im März 1166 stellt die Ausnahme von der Regel dar. Und auch dort war die kirchenrechtliche Problematik präsent. Aber auch nach der endgültigen Wiedervereinigung Kastiliens und Leóns 1230 ist es zu keinerlei konziliarer Tätigkeit vor Ort gekommen. Natürlich liesse sich klagen, dass es dem Papsttum und seinen Legaten nicht gelungen war, die regelmäßige Abhaltung von Provinzialkonzilien in Kastilien durchzusetzen. ${ }^{14}$ Auf der anderen Seite kam es eben auch im 13. Jahrhundert nicht zu einem Anknüpfen an die westgotische Konzilstradition, wie sie ganz ähnlich noch Anfang des 11. Jahrhunderts praktiziert worden war. Es kam zu keinen reichsweiten Konzilien ohne päpstliche Erlaubnis bzw. ohne die Anwesenheit eines päpstlichen Legaten. Auch wenn dies an keiner Stelle in den Quellen explizit angesprochen wird, darf man dem Papsttum in dieser Frage ein ganz spezielles Interesse unterstellen, denkt man an die stark durch ihre selbstständige Konzilstätigkeit charakterisierte, vom Papst-

14 Vgl. Linehan, Church, S. 35f. 
tum weitgehend abgekoppelte westgotische Kirche. Zu Beginn des 12. Jahrhunderts war der kastilische Episkopat außerdem trotz Aufforderung nicht auf die durch das Papsttum geleiteten Konzilien nach dem Norden der Pyrenäen gereist. Ab 1123 jedoch nahm regelmäßig eine beachtliche Anzahl von Klerikern aus der Kirchenprovinz Toledo an den vom Papst geleiteten Konzilien teil, so etwa 1123 und 1139 im Lateran, 1148 in Reims, 1163 in Tours oder 1179 wiederum im Lateran. Es lässt sich damit feststellen, dass die konziliare Tätigkeit vor Ort zurückging - eine derartig hohe Frequenz an Kirchenversammlungen wie in den ersten beiden Jahrzehnten des 12. Jahrhunderts sollte es in Kastilien und León danach nicht mehr geben - und der Toledaner Klerus stattdessen in wachsender Anzahl und Häufigkeit auf die päpstlich geleiteten Konzilien mit universalem Anspruch zog. Diese Entwicklungen stellen zweifelsfrei einen der radikalsten Brüche mit der Westgotenzeit dar. Gleichzeitig zählen sie in Bezug auf eine Integration des Untersuchungsraums in die durch das Papsttum gelenkte Gesamtkirche zu den wichtigsten Erfolgen.

Trotz eindeutig ablesbarer Tendenzen zur Verrechtlichung verdrängten diese allerdings nicht die Bedeutung persönlicher Kontakte zum päpstlichen Hof, um die eigenen Ziele zu erreichen. Als Papst Urban II. 1088 die Primatswürde Toledos bestätigte, hatte dies den Charakter der Wiedererrichtung eines alten Rechtsstands. Und Papst Lucius III. teilte 1182 dem Toledaner Kathedralkapitel mit, dass er trotz seiner Sympathie für Toledo und des Einsatzes des Kardinalpriesters Pedro de Cardona von S. Laurentius in Damaso die Privilegien des Santiagoordens nicht einfach ohne die einstimmige Zustimmung seines Kardinalskollegiums kassieren könne. Vorhandene Rechtstitel waren nicht einfach ignorierbar, weder zu Beginn noch am Ende des Unteruchungszeitraums. Je mehr Privilegien allerdings die Päpste an immer mehr Empfänger ausstellten, desto enger wurde der Rahmen, um flexibel handeln zu können. Trotzdem prägten persönliche Bekanntschaften, Antipathien und sicherlich häufig durch Geld geschaffene Verbindlichkeiten gerade im für die päpstliche Entscheidungsfindung immer wichtiger werdenenen Kardinalskolleg weiterhin die Kommunikation mit dem Papsttum. Bereits die Privilegien von 1088 und 1093 lassen sich kaum ohne ihren situativen Rahmen und die gemeinsame Vorgeschichte von Erzbischof Bernhard und Papst Urban II. erklären. Ähnlich nachhaltig wirkte sich die persönliche Gegnerschaft zwischen Bernhard und Richard von Marseille bzw. Johannes von Gaeta aus. Zwar konnte selbst Gelasius II. 1118 Bernhard von Toledo die Bestätigung des Toledaner Primats nicht verweigern, das Privileg allerdings stellte durchaus kein Ruhmesblatt für Erzbischof Bernhard dar und besaß weit eher den Charakter eines Mahnschreibens. Erzbischof Raimund erscheint in den Quellen wiederum um so vieles blasser als sein energischer Vorgänger. Selbst im Primatsstreit wurde seine Passivität deutlich. Dass sich der Kö- 
nig in den 1130er und 1140er Jahren so stark auf päpstliche Legaten a latere stützte und Erzbischof Raimund kaum hervortrat, dafür könnte ein Grund durchaus auch in der wohl eher zurückhaltenden Persönlichkeit dieses Prälaten zu finden sein. Die persönliche Verbundenheit zwischen dem päpstlichen Kanzler Roland und Erzbischof Johannes, derer sich Papst Alexander III. zu Beginn des Schismas erinnerte, führte offensichtlich zur entschiedenen Unterstützung der alexandrinischen Position durch Erzbischof Johannes auf der Iberischen Halbinsel und zu großem Entgegenkommen des Papstes im Primatsstreit, selbst als Erzbischof Johannes in Pamplona Verhaltensweisen zeigte, die man eher von seinem Vorgänger Bernhard kannte (welcher aber wiederum mit großer Sicherheit nicht auf eine derartig milde päpstliche Reaktion hätte hoffen dürfen). Privilegien und Rechtsentscheidungen waren und blieben abhängig vom Geldbeutel und dem Verhandlungsgeschick des Petenten. Dies und die Tendenz zur Verrechtlichung stellten keinen Widerspruch dar, sondern griffen ineinander und ergänzten sich. So konnte Erzbischof Johannes bei Papst Hadrian IV. 1156 die Rücknahme der Exemtion Compostelas aus dem Toledaner Primat - zweifelsfrei eine Sternstunde Toledaner Verhandlungsgeschicks - den Worten des Papstes nach erreichen, da jene Exemtion nicht auf Zustimmung der Kardinäle und damit nicht auf formalrechtlich korrektem Weg zustande gekommen sei. Dass sich Erzbischof Johannes zur selben Gelegenheit jedoch so nachhaltig für die St-Rufianer Regularkanoniker in Kastilien einsetzte, und damit genau die persönlichen Vorlieben dieses Papstes traf, dürfte sich aber mindestens ebenso sehr auf die päpstliche Entscheidung ausgewirkt haben.

Die regionalen und lokalen Besonderheiten der Untersuchungsregion spielten in den hier analysierten Beziehungen zwischen kirchlichem Zentrum und geographischer Peripherie eine unterschiedlich große Rolle. Toledo blieb im 12. Jahrhundert eine Stadt, die am Rand des orbis christianus lag, den Angriffen der Almoraviden und Almohaden ausgesetzt war und als kultureller sowie religiöser Schmelztiegel gelten kann. Gerade Letzteres war allerdings in der Korrespondenz mit dem Papsttum kaum von Bedeutung. Am Beispiel des sogenannten ,altspanischen' Ritus wurde deutlich, dass auch die Christenheit selbst nicht als homogener Monolith gesehen werden darf, sondern durchaus heterogene Züge aufwies. Die Homogenisierung der liturgischen Unterschiede war eines der erklärten Ziele Papst Gregors VII., die Wichtigkeit der Ritusfrage ging nach dessen Tod allerdings offenbar stark zurück. Weder lassen sich tiefgreifende und lang anhaltende Konflikte zwischen den Anhängern des alten Ritus und dem Papsttum, noch mit dem aus dem späteren Frankreich stammenden Klerus nachweisen. Das diesbezügliche Mandat Eugens III. blieb eine Ausnahme. Ebensowenig griff das Papsttum in das Zusammenleben der unterschiedlichen Religionen ein. Verklausulierte Missionsaufrufe Urbans II. und die Auffor- 
derung Coelestins III., mehrsprachige Priester zu denjenigen Christen zu entsenden, die unter muslimischer Herrschaft lebten, sind allenfalls als Ansätze zu bezeichnen. Aus der Kirchenprovinz Toledo ist für das 12. Jahrhundert schließlich nicht bekannt, dass das Papsttum als Garant des rechten Glaubensvollzugs, als Institution in Glaubensfragen, jemals angerufen worden wäre, auch wenn besonders in der prächtigen Ausgestaltung und den formelhaften Wendungen der Papstprivilegien die Heiligkeit des Petrusnachfolgers präsent war. Was aber die militärische Situation betrifft, lässt sich durchaus ein päpstliches Eingehen auf lokale Besonderheiten ablesen. Dass die Erzbischöfe von Toledo zu provisorischen Metropoliten für alle Diözesen ernannt wurden, deren eigene Metropolen noch nicht wiedererrichtet worden waren, zeigt, dass die Dynamik der Reconquista in der Privilegierung der Toledaner Kirche von Anfang an eine Rolle spielte. Auch die Überlassung von Complutum (Alcalá de Henares) und die zumindest zeitweise Übertragung Segovias sowie des päpstlichen Eigenklosters San Servando ist als päpstliche Reaktion auf die militärische Lage vor Ort zu verstehen. Und sobald sich das Papsttum über seine Stellvertreter einen unmittelbaren Eindruck von der Situation vor Ort gemacht hatte, spielte Toledo eine wichtige Rolle in der päpstlichen Reconquistapolitik. Dass die westgotische Tradition in Toledo einen noch größeren Stellenwert besaß als anderswo auf der Iberischen Halbinsel, erklärt sich aus der ehemaligen Stellung Toledos als weltliches und kirchliches Zentrum des Westgotenreichs. War für Papst Gregor VII. eine Rückkehr zur verlorenen Einheit mit Rom und der iberische Neogoticismus miteinander noch vollkommen unvereinbar, bewirkte Urban II. eine gewisse Versöhnung beider Vorstellungen, indem er das Papsttum zu der Instanz erklärte, die bestätigte, was den westgotischen Verhältnissen entspreche und was nicht. Diese Versöhnung erwies sich in der Folge bezüglich Toledo jedoch als oberflächlich. Einige Aspekte, neben der westgotischen Konzilspraxis vor allem die beinahe papstähnliche Stellung, die sich aus der Westgotenzeit für den Toledaner Primas reklamieren ließ, waren weder für das Papsttum noch für den übrigen iberischen Klerus akzeptabel. Besonders daraus resultierte die Krise der Jahrhundertwende und die Reaktion der Gegner Toledos bestand zum einen in der konsequenten Zurückdrängung jeglichen Westgotenbezugs in den Toledaner Primatsprivilegien und zum anderen in der Forcierung der Restauration der übrigen iberischen Metropolen. Erst in der zweiten Hälfte des 12. Jahrhunderts wurde der Zusammenhang zwischen Toledaner Primat und westgotischer Vergangenheit wieder explizit in Papsturkunden aufgerufen. Mittlerweile allerdings war die Primatswürde inhaltlich nur noch auf einen Ehrenvorrang reduziert und derartig abhängig von päpstlicher Privilegierung, dass dies gefahrlos möglich war. Erst jetzt war das Papsttum mit der westgotischen Tradition gänzlich ausgesöhnt. Die westgotische Kirchenorganisation als Blaupause für die Gestaltung 
der Gegenwart wurde vom Papsttum niemals grundsätzlich abgelehnt. Schrittweise wurde sogar jenes aus der Kirchenproninz Toledo stammende Machwerk, dessen Fälschungscharakter Anfang des 11. Jahrhunderts sogar bescheinigt worden war, die Divisio Wambae, vom Papsttum als authentisches Dokument anerkannt. Die Kirchenprovinz Toledo war räumlich 1185 eine völlig andere als hundert Jahre früher. War das Bistum Palencia noch ohne päpstliche Beteiligung wiedererrichtet worden, wurde ihm durch die Palentiner Fälscher des 12. Jahrhunderts eine solche Beteiligung zugeschrieben, was bereits die gestiegene Bedeutung der Päpste bei der Errichtung bzw. Restauration von Bistümern und der generellen kirchlichen Raumgestaltung verdeutlicht. Das Bistum Osma wurde zu Beginn des 12. Jahrhunderts auf eine direkte Aufforderung Papst Urbans II. hin wiedererrichtet. Auf die krisenhafte Entfremdung mit dem Papsttum und die päpstliche Übertragung der Metropolitanwürde Méridas an Santiago de Compostela lassen sich die Initiativen Erzbischof Bernhards zurückführen, zu Beginn der 1120er Jahre eine ganze Reihe von Bistümern (Zamora, Segovia, Sigüenza) zu restaurieren, um den Mangel an eigenen Suffraganen auszugleichen. Zamora als Toledaner Suffraganbistum zu halten, scheiterte endgültig erst Mitte des Jahrhunderts, als Erzbischof Johannes von Braga die Forderung nach Obödienz gegenüber dem Toledaner Primat mit der Frage um Zamora verknüpfte. Die Exemtion der Bistümer Burgos, León und Oviedo, durch die das Papsttum diese wichtigen Diözesen noch enger an sich band, war auf Kosten Toledos erfolgt und verdrängte den Toledaner Erzbischof aus dem Norden der Iberischen Halbinsel. Papst Calixt II., dem es nicht ausschließlich um eine Förderung Compostelas, sondern allgemeiner um eine Förderung des Reichs seines Neffen Alfons VII. ging, ignorierte im November 1121 bewusst oder unwissentlich die Exemtion Oviedos und Leóns und zählte beide Diözesen nochmals unter die Suffragane Toledos. In diesem motivlichen Zusammenhang ist auch die Wiedererrichtung der Diözesen Segovia und Sigüenza zu verstehen, die im ersten Fall durch ein päpstliches Privileg sanktioniert und im zweiten Fall durch die Anwesenheit des päpstlichen Kaplans Bonetus beeinflusst wurde, auch wenn in beiden Fällen die weltlichen Herrscher die entscheidende Rolle einnahmen. Nicht nur Grenzstreitigkeiten mit Nachbardiözesen wurden schließlich mehr und mehr am päpstlichen Gericht oder auf Legatenkonzilien entschieden. Die Bischöfe der Toledaner Suffraganbistümer bemühten sich seit dem zweiten Jahrzehnt des 12. Jahrhunderts um päpstliche Schutzprivilegien für ihre Besitzungen und Grenzen. Bei der Errichtung eines Bistums in Albarracín 1172 wurde dann deutlich, dass die durch einen Trick oder in voller Kenntnis der Sachlage herbeigeführte Entscheidung eines päpstlichen Legaten den erklärten Willen des aragonesischen Königs und sogar die Papsturkunden des Bischofs von Zaragoza ausstach. Bei der Schaffung des Bistums Cuenca schließlich spielte der Eroberer 
Alfons VIII. offiziell überhaupt keine Rolle mehr, als der beteiligte Klerus einzig und allein das Papsttum als Entscheidungsinstanz über die Erlaubnis zur Zusammenlegung zweier alter Bistümer und die Errichtung eines Kathedralkapitels anriefen. In keinem bekannten Fall wurden in der Kirchenprovinz Toledo eventuell noch existierende autochtone christliche Strukturen bzw. Organisationsformen päpstlicherseits anerkannt oder waren überhaupt nur der Erwähnung wert. Das Papsttum war somit ganz entscheidend an der Entstehung der Kirchenprovinz Toledo beteiligt, über den Untersuchungszeitraum von rund hundert Jahren gelang die Schaffung einer hierarchisch auf Rom hin orientierten Kirchenstruktur. Während dieses Prozesses wurde den römischen Päpsten immer mehr Autorität zugesprochen, die diese umgekehrt immer häufiger in Anspruch nahmen. Als weitere lokale Besonderheit der Diözese Toledo lässt sich schließlich anführen, dass in der Korrespondenz mit dem Papsttum kaum monastische Einrichtungen greifbar werden. Es stellt sich die Frage, inwieweit stattdessen nicht die Ritterorden in ihrer Verbindung von Mönch- und Kriegertum das klassische Mönchtum an der umkämpften Grenze zum muslimischen Süden der Halbinsel ersetzten. Zwar bezogen die Päpste in den zahlreichen Streitigkeiten, die sich zwischen den Orden und der lokalen Kirchenhierarchie entwickelten, nicht automatisch Position für die Ordensritter, Rom hatte ihnen jedoch weitreichende Exemtionsprivilegien gewährt und sie damit, nicht unähnlich exemierter Klöster, direkt dem hl. Stuhl unterstellt. Gerade in der zweiten Hälfte des 12. Jahrhunderts übernahmen die Ritterorden die Sicherung weiter Grenzbereiche im Süden der Diözese Toledo gegen die Almohaden. Dabei ist nicht feststellbar, dass die Päpste die Ritterorden zum Ausbau ihrer Autorität vor Ort genutzt oder in die durch die Orden organisierten Kirchenstrukturen eingegriffen hätten, wenn sie nicht aufgrund von Streitigkeiten mit der Diözesanhierarchie darum angerufen wurden. Schließlich soll auch das Toledaner Kathedralkapitel längere Zeit durch Kleriker aus cluniazensischem Umfeld geprägt worden sein. Wie monastisch das Kathedralkapitel zur Zeit Erzischof Bernhards tatsächlich organisiert war, lässt sich schwer feststellen, Toledo war allerdings in den 30er Jahren des 12. Jahrhunderts noch als Monolith des alten benediktinischen Mönchtums wahrnehmbar und die Region wies eine im Vergleich zu anderen Gegenden der Iberischen Halbinsel deutliche Verspätung bei der Etablierung der Augustinusregel in der Kirchenprovinz auf. Interessanterweise fiel die Öffnung einiger Suffraganbistümer für das neue Regularkanonikertum häufig mit der Präsenz päpstlicher Legaten oder dem Besuch päpstlich geleiteter Konzilien zusammen. Ein Einfluss des Papsttums bei der Öffnung der Kirchenprovinz für die Augustinusregel darf somit angenommen werden. Und um die Jahrhundertmitte erfuhr die Kirchenprovinz Toledo einen Generationenwechsel, als der dem Regularkanonikertum aufgeschlossene Johannes von Segovia auf den Toledaner 
Erzstuhl gewählt wurde und in Sigüenza und Osma Männer wie Cerebrun und Johannes den Bischofstab ergriffen. Danach konnte sich nicht nur in Toledo selbst die Augustinusregel etablieren, man vermochte diese Neuausrichtung ab 1156 auch wirkungsvoll beim Papsttum einzusetzen.

Mit einigem Recht lässt sich sagen, dass die Kirche der Kirchenprovinz Toledo gegen Ende des Untersuchungszeitraums ein gutes Stück weit Teil der dem Anspruch nach allein durch das Papsttum geleiteten Universalkirche geworden war. Was diesen päpstlichen Leitungsanspruch anging, sah dies im Verhältnis zu den weltlichen Mächten des Untersuchungsraums allerdings anders aus. Päpstliche Schreiben an lokale Adelige, wie an die Lara-Grafen im Streit zwischen Osma und Sigüenza oder an den Navarresen Pedro Ruiz de Azagra stellten die Ausnahme dar. Der Adel wurde, falls anwesend, auf den Legatenkonzilien zwar Zeuge der Verbreitung päpstlicher Vorstellungen und im Fall des Bischofs von Osma zum ausführenden weltlichen Arm des Papsttums ernannt. Die schmale Quellenbasis ist aber nicht ausreichend für grundsätzliche Befunde, und es ist nicht auszuschließen, dass für den lokalen Adel das Papsttum im 12. Jahrhundert im Grunde eine quantité négligeable darstellte. Die Könige von Kastilien und León fanden während des gesamten Untersuchungszeitraums in den Prälaten der Kirchenprovinz Toledo, allen voran in den Toledaner Erzbischöfen, wichtige Berater und hielten sich, je weiter die machtpolitische Zersplitterung der Iberischen Halbinsel voranschritt, desto häufiger im Untersuchungsraum auf. Alfons VI. von Kastilien-Leon erwies sich dabei wie alle seine Nachfolger als weitgehend unbestrittener Herrscher seiner Kirche. Ohne königliche Zustimmung kam kein Bischof ins Amt und ein Bischof war und blieb königlicher Amtsträger. Kaum ein Papstprivileg an Toledo wurde ohne königliche Empfehlungsschreiben erworben. Auf den Konzilien spielten sie eine wichtige Rolle und auch Alfons VIII. hatte etwa im Konflikt um die Kanoniker von Valladolid unter Beweis gestellt, dass er nicht dazu bereit war, Entscheidungen, die ohne sein Wissen, geschweige denn gegen seinen Willen getroffen wurden, zu akzeptieren. Und das Papsttum hat diese Haltung niemals ernstlich in Frage gestellt. Es wirft ein bezeichnendes Licht auf das herrscherliche Selbstverständnis, wenn Alfons VII. zur Jahrhundertmitte feststellte: mihi a deo et a sede apostolica in penitentia et in remissione peccatorum meorum commissum est ut ecclesias dei in justitia regam. ${ }^{15}$ Alfons VII. sah also keinen Grund, sich in Konkurrenz zum päpstlichen Führungsanspruch zu wähnen, er sah sich vielmehr von Gott und dem Papst zur Kirchenherrschaft legitimiert. Das heißt jedoch nicht, dass nicht auch für weltliche Herrscher päpstliche Vorstellungen spürbar geworden wären. Ohne

15 Reg. Reilly, Alfonso VII, D 660 (25.04.1150), S. 373; Druck Minguella y Arnedo (Ed.), Historia, Nr. 31, S. 386; vgl. auch Linehan, History, S. 250. 
die dezidierte Verurteilung des altspanischen Ritus durch Gregor VII. ist der Liturgiewechsel Ende des 11. Jahrhunderts in Kastilien-León schlichtweg nicht vorstellbar. Bei der Wiedereinsetzung des Diego Peláez von Compostela erwies sich Urban II. allerdings, der doch in seinem Schreiben Duo sunt rex gegenüber Alfons VI. deutlich gemacht hatte, welche Macht in gelasianischer Tradition die höhere sei, als weit weniger erfolgreich. Die Ehetrennung Urracas und Alfons' I. von Aragón war zwar auch in weiten Teilen besonders des galicischen Adels ein erklärtes Ziel, jedoch auch das Papst Paschalis' II. Sogar in Zeiten überbordendem königlichen Selbstbewusstseins, das seinen Höhepunkt in der Kaiserkrönung 1135 erfuhr, erwies sich Papst Innozenz II. als erfolgreicher Verteidiger päpstlicher Prärogativen bezüglich der exemten Bistümer in León und Kastilien. Der selbsternannte Kaiser akzeptierte die päpstlichen Vorstellungen zur Konzilienpraxis vor Ort und betrachtete den Papst und dessen Legaten viel eher als Kooperationspartner denn als Fremdkörper. Das hinderte ihn allerdings nicht daran, Papst und Legaten den herrscherlichen Zorn spüren zu lassen, wenn diese anders handelten, als er es wünschte. Zwar löste Alfons VII. den Primatsstreit aus, als er sich aber vor seinem Tod schließlich frustriert vom Papsttum abwandte, ging der Toledaner Episkopat diesen Weg nicht mit und focht weiterhin beim Papsttum für die Toledaner Privilegien. Womöglich lassen sich hier erste Ansätze einer Emanzipation von der nahezu totalen königlichen Kontrolle ablesen. Auch Alfons VIII. griff in die Belange seiner Kirche ein, wenn es ihm beliebte. Die Eroberung von Cuenca beispielsweise wurde wohl zu einem Großteil mit kirchlichen Geldern finanziert. Dabei scheute der König nicht davor zurück, Bestechungsgelder für seine Zustimmung zur Bischofswahl in Osma anzunehmen. Andererseits beeinflusste nicht nur das päpstliche Urkundenwesen das seine. Als der kastilische Episkopat auf das III. Lateranum hin geschlossen die königliche Bestätigung der Freiheitsrechte und der kirchlichen Unverletzlichkeit einforderte und damit besagte Emanzipationstendenzen fortsetzte, gewährte der König, was er der Kirche an Freiheiten schulde. Neben der zweiten Legationsreise Hyacinths sind dafür vor allem bestimmte Entwicklungen der krisenhaften 60er Jahre des 12. Jahrhunderts verantwortlich zu machen. Als König Ferdinand II. über das Bistum Osma seine Herrschaft auf Kastilien ausdehnen wollte, kam es zur direkten Konfrontation mit Papst Alexander III. in der Frage, wer von beiden in kirchlichen Angelegenheiten die höchste Entscheidungsgewalt habe. Mit Hilfe der antileonesichen Opposition in Kastilien setzte sich damals die päpstliche Position durch. Ferdinand II. musste nicht nur zusehen, dass sich seine Forderung, dass der Papst nichts umzustoßen hätte, was er selbst rechtmäßig entschieden habe, angesichts der Verhältnisse in Kastilien nicht durchsetzen und dass sich der Papst mehr zum Unterstützer der selbständigen Herrschaft Alfons VIII. machen ließ. Die Quellen dazu, dass sich ein durch ein Schisma geschwächtes Papsttum 
in zwei grundlegenden Aspekten der Kirchenreform, in der Frage des Eingriffs weltlicher Mächte in kirchliche Angelegenheiten und schließlich auch in der Frage um die Simonie in Osma durchsetzen konnte, lassen diese Ereignisse allerdings weitgehend als ein singuläres Ereignis erscheinen.

Ohne Zweifel hatte das Papsttum in den hundert Jahren zwischen 1085 und 1185 in der Kirchenprovinz Toledo erfolgreich Integrationsprozesse angestoßen und war die eingangs von Bernhard Peters zitierten drei Problembereiche angegangen. In Form zahlreicher Grenz- und Rechtsentscheidungen hatte es in die objektive Welt eingegriffen, und durch die Verbreitung kirchenreformerischer und kirchenrechtlicher Vorstellungen auf Konzilien sowie durch Rechtssammlungen wurde dem lokalen Klerus Orientierung gegeben und die Ausbildung von Wertmaßstäben angeregt. Repräsentative Akte wie die Übertragung des Palliums oder der Mitra oder auch die Angleichung der Gottesdienstordnungen förderten die Ausbildung kollektiver Identität unter dem Toledaner Klerus als Teil einer Gesamtkirche. Und indem sich das Papsttum als neutrale und zuverlässige Gerichtsinstanz etablierte, sorgte es für den Ausgleich divergierender Interessen und konfligierender Ansprüche. Kein Toledaner Prälat konnte am Ende des 12. Jahrhunderts mehr daran zweifeln, dass der Papst in Rom (wenigstens theoretisch) Herr der Gesamtkirche sei. Diese Position war insofern unbestritten, als dass er Rechtsstände legitimierte, beurkundete und beschützte und Streitfälle am päpstlichen Gericht oder vor Ort durch delegierte Richter oder Legaten entscheiden ließ. Die gewachsene Autorität des Papsttums wurde besonders in der Anerkennung seiner Schiedsrichter- und Ordnungsfunktion deutlich. Dabei griff das Papsttum selten von sich aus in die Verhältnisse vor Ort ein, sondern wurde von den lokalen Kräften angerufen. Von ihnen wurde dem Papsttum mehr und mehr Bedeutung zugeschrieben und Entscheidungsgewalt zugestanden. Von der Möglichkeit allerdings, ganz selbstverständlich „,den eigenen Willen auch gegen Widerstreben“" vor Ort durchzusetzen, was Max WEBER als entscheidenden Faktor von Machtausübung begreift, ${ }^{16}$ davon konnte für das Papsttum auch am Ende des 12. Jahrhunderts kaum die Rede sein.

16 Vgl. Max Weber, Wirtschaft und Gesellschaft, Bd. 1. Tübingen ${ }^{5} 1976$, S. 28, Zitat ebendort; Michael Mann, Geschichte der Macht. Bd. 1: Von den Anfängen bis zur griechischen Antike. Frankfurt / New York 1990, S. 22. 